(C) 2019 IEEE

\title{
Unidirectional High-Power DC-DC Converter Utilizing Scott Transformer Connection
}

S. Milovanovic and D. Dujic

This material is posted here with permission of the IEEE. Such permission of the IEEE does not in any way imply IEEE endorsement of any of EPFL's products or services. Internal or personal use of this material is permitted. However, permission to reprint / republish this material for advertising or promotional purposes or for creating new collective works for resale or redistribution must be obtained from the IEEE by writing to pubs-permissions@ieee. org. By choosing to view this document, you agree to all provisions of the copyright laws protecting it. 


\title{
Unidirectional High-Power DC-DC Converter Utilizing Scott Transformer Connection
}

\author{
Stefan Milovanović and Dražen Dujić \\ Power Electronics Laboratory - PEL \\ École Polytechnique Fédérale de Lausanne - EPFL \\ Station 11, CH-1015 Lausanne \\ stefan.milovanovic@epfl.ch,drazen.dujic@epfl.ch
}

\begin{abstract}
This paper proposes unidirectional, isolated, DC-DC converter intended to act as an interface between high/medium voltage bipolar DC grid (or two separate DC grids) and another DC grid of an arbitrary voltage level. With the aim of achieving galvanic isolation, along with voltage matching, Scott transformer connection is employed. Furthermore, medium frequency operation of the isolation stage is considered, leading to an increase in converter's power density. The proposed converter combines two series connected full-bridge modular multilevel converters, within the high/medium voltage stage, and conventional threephase diode rectifier, within the low voltage stage, by means of the Scott transformer connection. In order to present basic operating principles and evaluate the proposed converter performance, simulation results of $40 \mathrm{kV} / 1.5 \mathrm{kV}, 10 \mathrm{MW}$ system are presented throughout this paper.
\end{abstract}

\section{INTRODUCTION}

Conversion of the existing AC power lines into DC has been recognized as a feasible approach to the question of increasing power capacity of the currently existing power system [1]-[4]. While both unipolar and bipolar configurations are possible, bipolar DC grids with neutral conductor offer the possibility of exploiting the so-called redundancy principles [3], [4]. Under normal operating conditions, neutral conductor does not carry any current (ideally). However, should any of the DC feeders malfunction, neutral conductor can overtake its role, thus enabling the system to continue the operation. Nevertheless, certain system reconfiguration or operating mode change might be required on these terms. Fig. 1a depicts generalized structure of an isolated DC-DC converter to be employed within a bipolar grid with neutral conductor. Part of the converter interfacing the grid comprises two independent units, both delivering half the rated power to the isolation stage under normal operating conditions. If either of the DC poles is lost, the converter being associated to it gets disconnected from the rest of the circuit, allowing the system to continue the operation although with reduced power, as presented in Fig. $1 b$.

Even though significant amount of research has been conducted in the field of non-isolated DC-DC converters [5]-[8], isolation is still considered mandatory within most of the applications due to safety reasons. Frequencywise, operation of a DC-DC converter is not limited to the line frequency $(50 / 60 \mathrm{~Hz})$, therefore bulky Low Frequency Transformer (LFT) can be replaced by more compact Medium Frequency Transformer (MFT), resulting in volumetric and gravimetric power density increase.

This paper proposes a novel unidirectional, high power, DC-DC converter utilizing Scott Transformer Connection (STC), operating in the medium frequency range, with the aim of achieving galvanic separation between two DC grids of different voltage levels. In order to interface High Voltage (HV)/Medium Voltage (MV) at either of the converter stages, series connection of two Single-Phase (1PH) Modular Multilevel Converters (MMCs) [9] is used, whereas conventional Three-Phase (3PH) Diode Rectifier (DR) is employed at the Low Voltage (LV) stage. Therefore, this paper

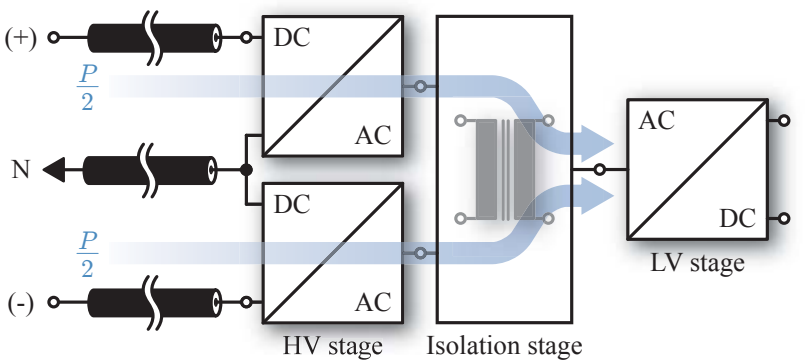

(a)

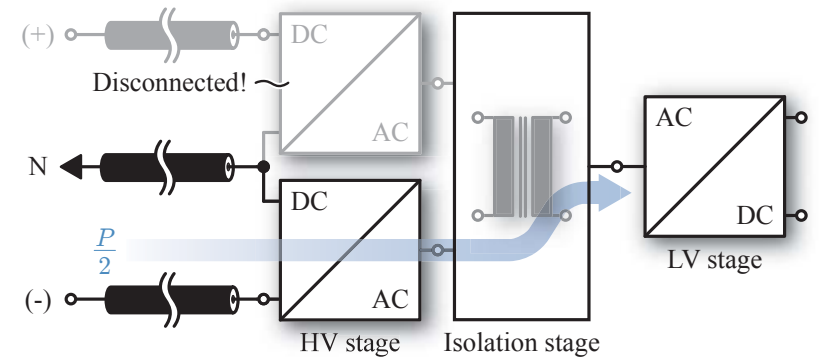

(b)

Figure 1: Generalized structure of a converter to be employed within a bipolar DC grid; a) Normal operating conditions. Both DC-AC converters supply the isolation stage with half the rated power. b) Converter operation in case positive DC is lost. Converter being connected to the faulty pole gets isolated from the circuit, therefore the system continues to operate with half the rated power. Please notice that, on these terms, neutral conductor overtakes the role of the pole being lost. Also, the same reasoning can be applied in case negative pole is lost. 
analyses variation of the topology presented in [10], focusing upon the new control methods adjusted for the needs of unidirectional power transfer.

The predominant use of the STC concerns the railway applications, implying low frequency operation. It is primarily used with the aim of obtaining two $1 \mathrm{PH}$ voltage sources out of a symmetrical $3 \mathrm{PH}$ voltage source. Additionally, employment of the MMC in the railway applications was studied in [11]-[14]. The possibility to employ the so-called direct MMC in order to provide a single railway line with sinusoidal voltage was analyzed in [11], whereas the MMC having a role of the traction converter, was studied in [12]. In order to obtain a railway power conditioner, being in charge of the railway grid STC currents balancing along with harmonics compensation, utilization of two back-to-back 1PH-MMCs was proposed in [13]. The STC employment has also been reported in [15]-[18] with the aim of obtaining the unity power factor rectifier. Nevertheless, analyzed power, operating frequency and voltage ranges fall significantly bellow the one being the focus of this paper. Additionally, neither of the references [11]-[18] has considered combining the MMC with the STC in order to perform the DC-DC conversion. Consequently, the operating frequency shift, which reflects positively upon the system size implying reduced material requirements, is allowed.

This paper proposes joint operation of the STC and the 3PH DR, in the field of high power DC-DC conversion, for the first time. Hence, its main original contributions can be summarized as:

- A novel unidirectional high-power DC-DC converter utilizing the STC is proposed

- Medium frequency operation of the STC is proposed

- A novel topology allowing redundancy principles, inherently present within bipolar grids with neutral conductor, to be exploited is presented
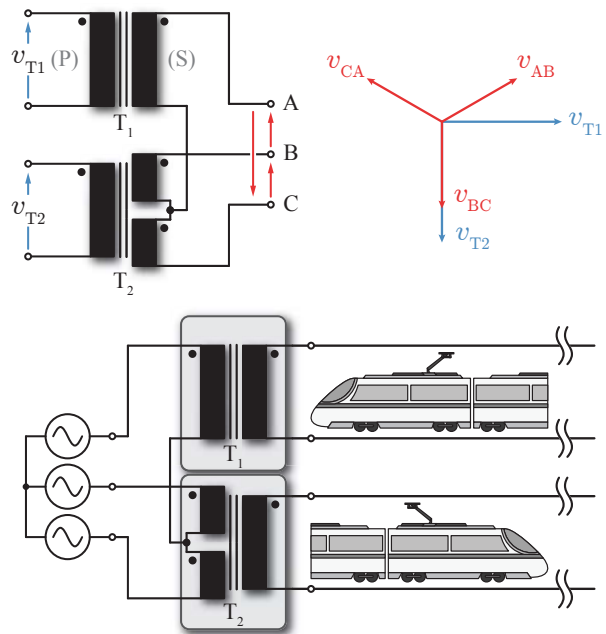

Figure 2: The STC with its voltage phasor diagram (upper); Common employment of the STC (lower) - two 1PH voltage sources are obtained from a symmetrical $3 \mathrm{PH}$ grid with the aim of supplying two separate railway lines.

\section{ThE PROPOSED TOPOLOGY}

Fig. 2 presents the STC with its voltage phasor diagram. It comprises two $1 \mathrm{PH}$ transformer units $\left(\mathrm{T}_{1}\right.$ and $\left.\mathrm{T}_{2}\right)$, with one of them $\left(\mathrm{T}_{2}\right)$ being center-tapped. With $\mathrm{T}_{2}$ Secondary (S)-winding number of turns being denoted by $N$, to ensure the proper STC operation, $\mathrm{T}_{1} \mathrm{~S}$-winding number of turns has to be set as $N \sqrt{3} / 2$. With the equal number of Primary $(\mathrm{P})$-winding number of turns, $\mathrm{T}_{1}$ and $\mathrm{T}_{2}$ turns ratios $\left(m_{\mathrm{T} 1}\right.$ and $m_{\mathrm{T} 2}$, respectively) relation is $m_{\mathrm{T} 1}=\frac{2}{\sqrt{3}} m_{\mathrm{T} 2}$. Moreover, both transformers' P-winding voltages can be expressed by means of the STC S-side quantities (1).

$$
\begin{aligned}
& v_{\mathrm{T} 1}=m_{\mathrm{T} 1} \frac{v_{\mathrm{AB}}-v_{\mathrm{CA}}}{2} \\
& v_{\mathrm{T} 2}=m_{\mathrm{T} 2} v_{\mathrm{BC}}
\end{aligned}
$$

As already stated, the STC has mostly been used within the railway applications with the aim of obtaining two $1 \mathrm{PH}$ voltage sources out of a symmetrical $3 \mathrm{PH}$ voltage source, as shown in Fig. 2. Hence, two separate railway lines can be supplied out of one $3 \mathrm{PH}$ voltage source. On the other hand, one can notice that in case two $1 \mathrm{PH}$ sinusoidal voltages, shifted with respect to each other by $90^{\circ}$, are applied across $\mathrm{T}_{1}$ and $\mathrm{T}_{2} \mathrm{P}$-windings, symmetrical system of line voltages will be observed from the $\mathrm{S}$-side. Following the aforementioned analysis, reference [10] proposed high-power, bidirectional, DC-DC converter utilizing the STC to achieve galvanic isolation between two conversion stages. Operating principles relied on the ones being presented with the appearance of the Dual-Active Bridge (DAB) [19]. However, if unidirectional power flow is required, conventional Six-Step Converter (SSC) employed at the LV stage, can be replaced by the $3 \mathrm{PH} \mathrm{DR}$, leading to the topology presented in Fig. 3.

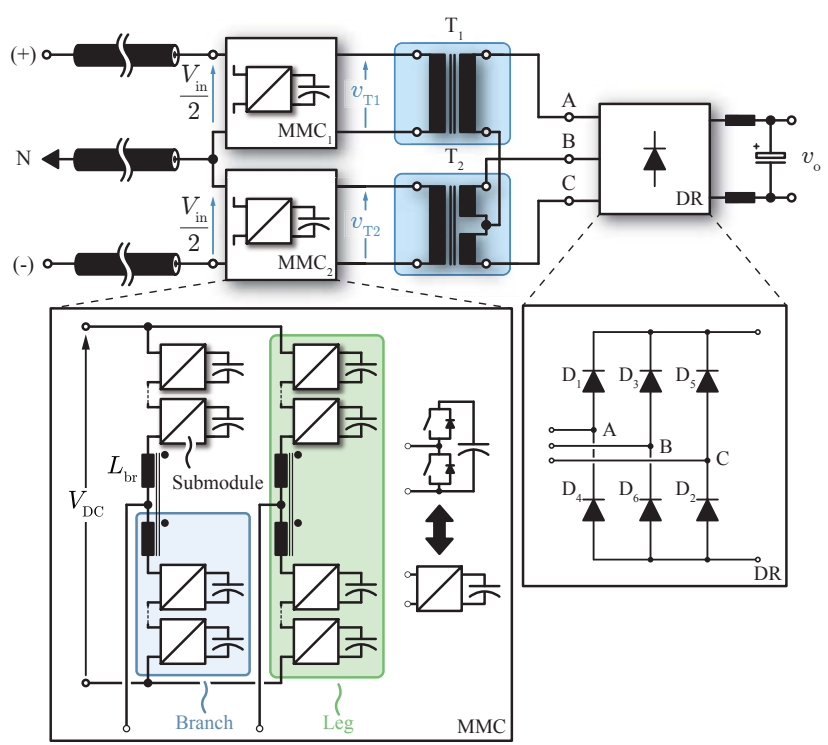

Figure 3: The proposed topology; Two FB MMCs are used in order to interface HV/MV bipolar grid. By virtue of the STC, galvanic separation between two different grids is achieved. Considering the converter is intended to provide the means for unidirectional power flow, 3PH DR is employed at the LV stage. 
With the aim of interfacing HV/MV bipolar grid, two series connected Full-Bridge (FB) MMCs employing HalfBridge (HB) submodules (SMs) are employed. Number of SMs employed depends upon the available DC voltage, as well as the voltage class of semiconductor devices to be used. The proposed topology operates with branch inductors being coupled, with the purpose which is going to be explained shortly. Last but not least, employing two separate transformer units evades any circulating currents between HV/MV converter stages, consequently having a positive effect on converter's susceptibility to parameters and generated waveforms mismatches.

\section{Preliminary considerations}

Fig. 4 presents the equivalent circuit of the 1PH MMC where clusters of SMs were replaced with the ideal voltage sources [20]. Additionally, branch inductors belonging to a same leg are coupled, therefore mutual inductance is denoted by $L_{\mathrm{m}}$. Two Kirchhoff Voltage Loops (KVLs), labeled with green and red, can be formed, leading to:

$$
\begin{gathered}
v_{\mathrm{ab}}=v_{\mathrm{pb}}-v_{\mathrm{pa}}+L_{\mathrm{br}} \frac{d i_{\mathrm{pb}}}{d t}+R_{\mathrm{br}} i_{\mathrm{pb}}-\ldots \\
\ldots-L_{\mathrm{br}} \frac{d i_{\mathrm{pa}}}{d t}-R_{\mathrm{br}} i_{\mathrm{pa}}-L_{\mathrm{m}} \frac{d i_{\mathrm{na}}}{d t}+L_{\mathrm{m}} \frac{d i_{\mathrm{nb}}}{d t} \\
v_{\mathrm{ab}}=v_{\mathrm{na}}-v_{\mathrm{nb}}+L_{\mathrm{br}} \frac{d i_{\mathrm{na}}}{d t}+R_{\mathrm{br}} i_{\mathrm{na}}-\ldots \\
\ldots-L_{\mathrm{br}} \frac{d i_{\mathrm{nb}}}{d t}-R_{\mathrm{br}} i_{\mathrm{nb}}+L_{\mathrm{m}} \frac{d i_{\mathrm{pa}}}{d t}-L_{\mathrm{m}} \frac{d i_{\mathrm{pb}}}{d t}
\end{gathered}
$$

where subscripts "p" and "n" denote upper and lower branch quantities, respectively. Summing (2) and (3), while keeping in mind that $i_{\mathrm{ab}}=i_{\mathrm{pa}}-i_{\mathrm{na}}=i_{\mathrm{nb}}-i_{\mathrm{pb}}$, yields (4).

$$
v_{\mathrm{ab}}=\frac{v_{\mathrm{na}}-v_{\mathrm{pa}}}{2}+\frac{v_{\mathrm{pb}}-v_{\mathrm{nb}}}{2}-\underbrace{\left(L_{\mathrm{br}}-L_{\mathrm{m}}\right)}_{L_{\sigma}} \frac{d i_{\mathrm{ab}}}{d t}-R_{\mathrm{br}} i_{\mathrm{ab}}
$$

One can notice that (4) can be further reduced in case the adjacent MMC legs operate in the complementary manner.

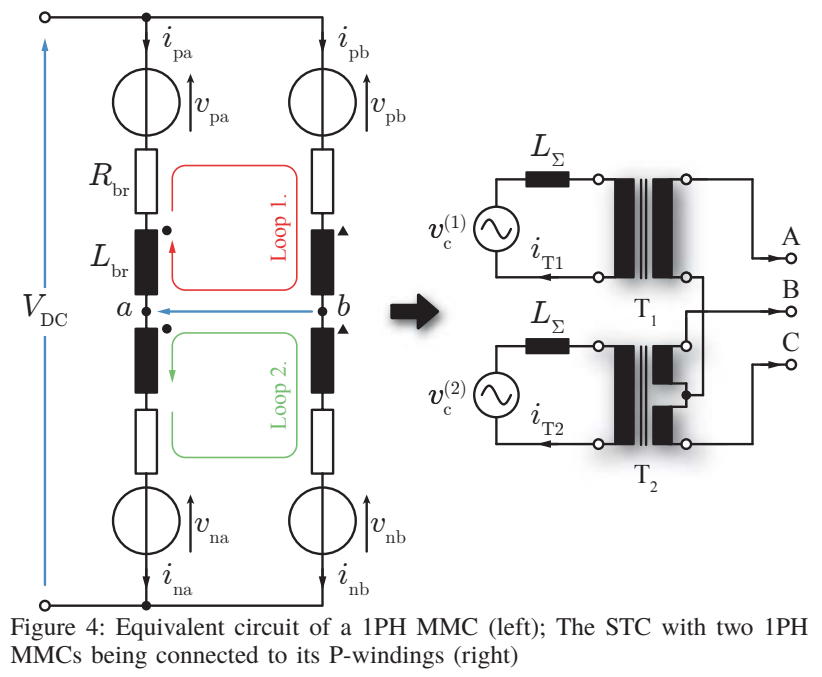

In other words, leg "b" voltages can be set as $v_{\mathrm{pb}}=v_{\text {na }}$ and $v_{\text {nb }}=v_{\text {pa }}$, which finally provides (5).

$$
v_{\mathrm{ab}}=\underbrace{v_{\text {na }}-v_{\mathrm{pa}}}_{v_{\mathrm{c}}}-L_{\sigma} \frac{d i_{\mathrm{ab}}}{d t}-R_{\mathrm{br}} i_{\mathrm{ab}}
$$

Consequently, seen from its $\mathrm{AC}$ terminals, the $1 \mathrm{PH} \mathrm{MMC}$ can be represented with the serial connection of voltage source $\left(v_{c}\right)$ and the equivalent MMC branch impedance $\left(L_{\sigma}=L_{\mathrm{br}}-L_{\mathrm{m}}\right)$, as presented on the right-hand side of Fig. 4. Sum of the MFT leakage inductance and equivalent MMC branch inductance was denoted by $L_{\Sigma}$. However, if the MFT leakage inductance can be considered negligible with respect to the equivalent MMC branch inductance, then $L_{\Sigma} \approx L_{\sigma}$. In order to establish a solid foundation for the upcoming system control related discussions, behavior of the STC S-side quantities needs to be investigated. Ampere-turns balance equations can be set for both transformer units, leading to (6).

$$
\left[\begin{array}{c}
i_{\mathrm{A}} \\
i_{\mathrm{B}} \\
i_{\mathrm{C}}
\end{array}\right]=\left[\begin{array}{cc}
m_{\mathrm{T} 1} & 0 \\
\frac{-m_{\mathrm{T} 1}}{2} & m_{\mathrm{T} 2} \\
\frac{-m_{\mathrm{T} 1}}{2} & -m_{\mathrm{T} 2}
\end{array}\right]\left[\begin{array}{l}
i_{\mathrm{T} 1} \\
i_{\mathrm{T} 2}
\end{array}\right]
$$

Further, STC S-side voltage balance equations can be derived (7).

$$
\begin{aligned}
& v_{\mathrm{AB}}=\frac{v_{\mathrm{c}}^{(1)}}{m_{\mathrm{T} 1}}-\frac{v_{\mathrm{c}}^{(2)}}{2 m_{\mathrm{T} 2}}-\frac{L_{\sigma}}{m_{\mathrm{T} 1}} \frac{d i_{\mathrm{T} 1}}{d t}+\frac{L_{\sigma}}{2 m_{\mathrm{T} 2}} \frac{d i_{\mathrm{T} 2}}{d t} \\
& v_{\mathrm{BC}}=\frac{v_{\mathrm{c}}^{(2)}}{m_{\mathrm{T} 2}}-\frac{L_{\sigma}}{m_{\mathrm{T} 2}} \frac{d i_{\mathrm{T} 2}}{d t} \\
& v_{\mathrm{CA}}=-\frac{v_{\mathrm{c}}^{(1)}}{m_{\mathrm{T} 1}}-\frac{v_{\mathrm{c}}^{(2)}}{2 m_{\mathrm{T} 2}}+\frac{L_{\sigma}}{m_{\mathrm{T} 1}} \frac{d i_{\mathrm{T} 1}}{d t}+\frac{L_{\sigma}}{2 m_{\mathrm{T} 2}} \frac{d i_{\mathrm{T} 2}}{d t}
\end{aligned}
$$

Substitution of (6) into (7) results in (8), from which one can notice that the equivalent MMC branch inductance becomes transferred to the STC S-side as $L_{\gamma}^{*}=L_{\sigma} /\left(2 m_{\mathrm{T} 2}^{2}\right)$. Dividing the equivalent MMC branch inductance by square of $\mathrm{T}_{2}$ turns ratio points out that the STC is indeed analyzed from its S-side. Subsequent division by two suggests that two MMCs operate in parallel observed from the STC S-side. Additionally, three equivalent line voltages $\left(v_{\mathrm{AB}}^{\prime}, v_{\mathrm{BC}}^{\prime}\right.$ and $\left.v_{\mathrm{CA}}^{\prime}\right)$ can be identified.

$$
\begin{aligned}
& v_{\mathrm{AB}}=\underbrace{\frac{v_{\mathrm{c}}^{(1)}}{m_{\mathrm{T} 1}}-\frac{v_{\mathrm{c}}^{(2)}}{2 m_{\mathrm{T} 2}}}_{v_{\mathrm{AB}}^{\prime}}-\underbrace{\frac{L_{\sigma}}{2 m_{\mathrm{T} 2}^{2}}}_{L_{\gamma}^{*}} \frac{d i_{\mathrm{A}}}{d t}+\frac{L_{\sigma}}{2 m_{\mathrm{T} 2}^{2}} \frac{d i_{\mathrm{B}}}{d t} \\
& v_{\mathrm{BC}}=\underbrace{\frac{v_{\mathrm{c}}^{(2)}}{m_{\mathrm{T} 2}}-\frac{L_{\sigma}}{2 m_{\mathrm{T} 2}^{2}} \frac{d i_{\mathrm{B}}}{d t}+\frac{L_{\sigma}}{2 m_{\mathrm{T} 2}^{2}} \frac{d i_{\mathrm{C}}}{d t}}_{v_{\mathrm{BC}}^{\prime}} \\
& v_{\mathrm{CA}}=\underbrace{-\frac{v_{\mathrm{c}}^{(1)}}{m_{\mathrm{T} 1}}-\frac{v_{\mathrm{c}}^{(2)}}{2 m_{\mathrm{T} 2}}}_{v_{\mathrm{CA}}^{\prime}}-\frac{L_{\sigma}}{2 m_{\mathrm{T} 2}^{2}} \frac{d i_{\mathrm{C}}}{d t}+\frac{L_{\sigma}}{2 m_{\mathrm{T} 2}^{2}} \frac{d i_{\mathrm{A}}}{d t}
\end{aligned}
$$



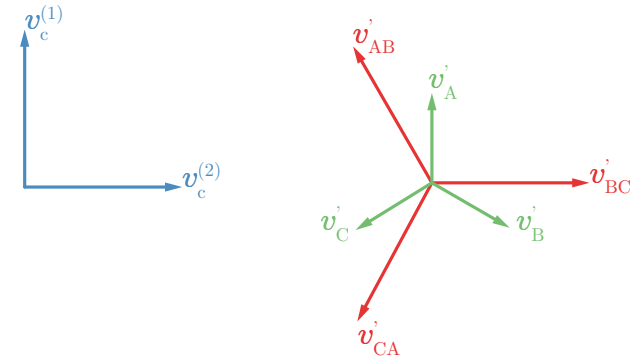

(a)

(b)

Figure 5: a) Two AC voltages generated by the MMCs interfacing the bipolar grid; b) Phasor diagram of an equivalent line voltage system seen from the STC S-side (red) along with its equivalent star (green)

In case sinusoidal voltages $v_{\mathrm{c}}^{(1)}$ and $v_{\mathrm{c}}^{(2)}$ are created by two MMCs interfacing the bipolar grid, their complex representatives can be chosen as $\vec{v}_{\mathrm{c}}^{(2)}=\vec{v}$ and $\vec{v}_{\mathrm{c}}^{(1)}=j \vec{v}$. Consequently, equivalent voltages seen from the STC S-side $\left(v_{\mathrm{AB}}^{\prime}, v_{\mathrm{BC}}^{\prime}\right.$ and $\left.v_{\mathrm{CA}}^{\prime}\right)$ can be described as (9). In case previously derived transformers' turns ratio relation holds, expression (9) describes a symmetrical system of line voltages. As depicted in Fig. 5, such a system of line voltages can be represented by the equivalent star of phase voltages $\left(\vec{v}_{\mathrm{A}}^{\prime}, \vec{v}_{\mathrm{B}}^{\prime}\right.$ and $\left.\vec{v}_{\mathrm{C}}^{\prime}\right)$.

$$
\begin{aligned}
& \vec{v}_{\mathrm{AB}}^{\prime}=\frac{\vec{v}}{m_{\mathrm{T} 2}}\left(-\frac{1}{2}+j \frac{m_{\mathrm{T} 2}}{m_{\mathrm{T} 1}}\right) \\
& \vec{v}_{\mathrm{BC}}^{\prime}=\frac{\vec{v}}{m_{\mathrm{T} 2}} \\
& \vec{v}_{\mathrm{CA}}^{\prime}=\frac{\vec{v}}{m_{\mathrm{T} 2}}\left(-\frac{1}{2}-j \frac{m_{\mathrm{T} 2}}{m_{\mathrm{T} 1}}\right)
\end{aligned}
$$

Consequently, the STC with two MMCs being connected to its P-windings can be perceived through the equivalent circuit from Fig. 6. It is straightforward to conclude that in case $3 \mathrm{PH}$ DR gets connected to the terminals A,B and C, no difference compared to the grid connected DR should be observed. Nevertheless, the above discussion refers to the normal operating conditions of the proposed converter. Should either of the DC poles be lost, operation of the converter can not be analyzed by means of the equivalent circuit from Fig. 6. On these terms, the converter operates as the 1PH DR, therefore new equivalent scheme is required. However, due to the lack of space, this paper presents the operation under

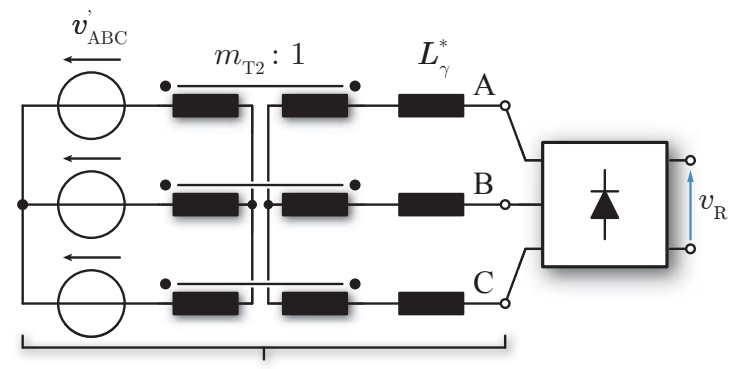

$\mathrm{STC}+$ two $1 \mathrm{PH}-\mathrm{MMCs}$

Figure 6: Equivalent circuit of the 3PH DR being connected to the STC along with two 1PH MMCs acting as a sinusoidal voltage sources normal operating conditions, leaving the question of operation under faults as a subject for future publications.

\section{OPERATING PRINCIPLES}

Discussion on the converter operating principles will be conducted by means of the $3 \mathrm{PH}$ equivalent from Fig. 6 . According to Fig. 5, the presence of two 1PH sinusoidal, quadrature, voltages $\left(v_{\mathrm{c}}^{(1)}\right.$ and $\left.v_{\mathrm{c}}^{(2)}\right)$ across $\mathrm{T}_{1}$ and $\mathrm{T}_{2} \mathrm{P}$-windings results in the symmetrical system of line voltages seen from the STC S-side $\left(v_{\mathrm{AB}}^{\prime}, v_{\mathrm{BC}}^{\prime}\right.$ and $\left.v_{\mathrm{CA}}^{\prime}\right)$. Further, according to (8)-(9), magnitude of the equivalent line voltages equals $\hat{v}_{s}=\hat{v}_{\mathrm{p}} / m_{\mathrm{T} 2}$, where $m_{\mathrm{T} 2}$ and $\hat{v}_{\mathrm{p}}$ designate transformer $\mathrm{T}_{2}$ turns ratio and voltage magnitude across both transformers' $\mathrm{P}$-windings, respectively. Consequently, with the voltage drop caused by the DR commutation being temporarily neglected, mean value of the DR output voltage equals $\bar{v}_{\mathrm{R}}^{*}=3 \hat{v}_{\mathrm{s}} / \pi$. Please notice that, by regulating the magnitude of voltages generated by the STC P-side MMCs, mean value of the DR output voltage can be adjusted. Fig. 3 shows that the standard $L C$ filter can be used to filter out the higher order harmonics from the DR output voltage. Therefore, DR output current can be approximated by its DC component, which allows the idealized operating waveforms of the converter to be presented as in Fig. 7.

To derive the complete expression for the converter output voltage mean value, $3 \mathrm{PH}$ DR commutation process must be

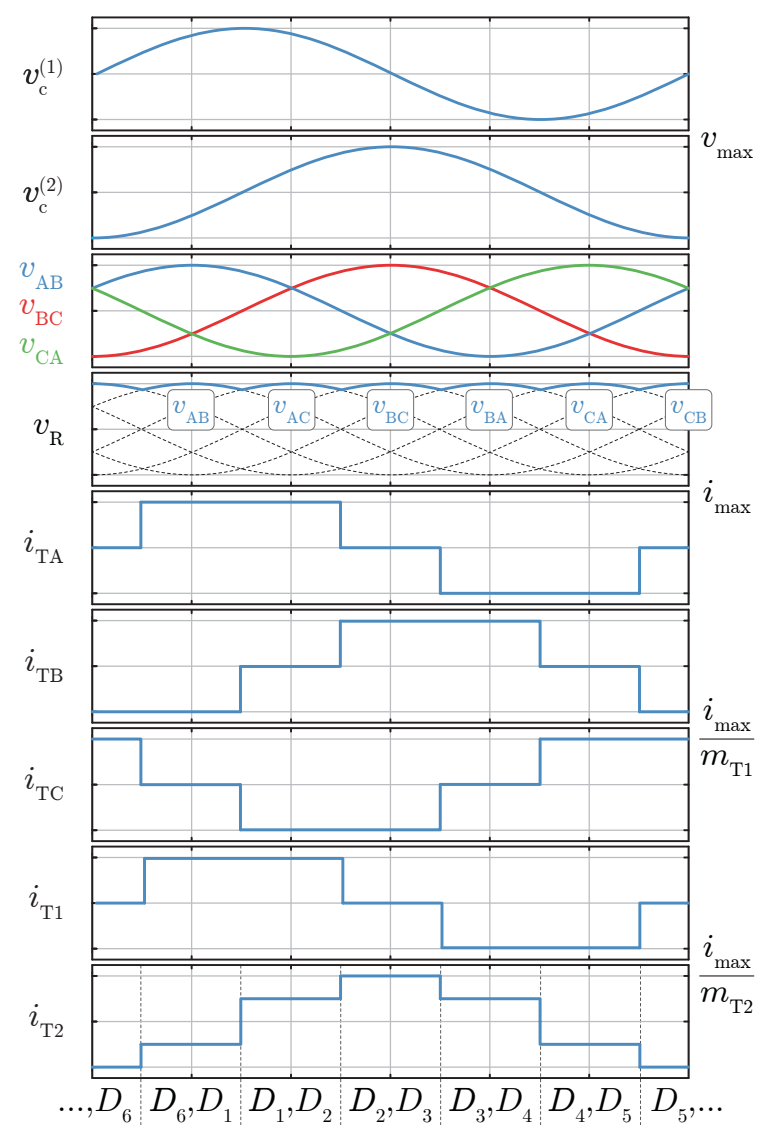

Figure 7: Converter relevant operating waveforms (idealized) 
Table I: Simulated system parameters

\begin{tabular}{lcc}
\hline \multicolumn{1}{c}{ Parameter } & Label & Value \\
\hline Input voltage & $V_{\text {in }}$ & $\pm 20 \mathrm{kV}$ \\
Output voltage & $V_{\mathrm{o}}$ & $1.5 \mathrm{kV}$ \\
Rated power & $P_{\text {nom }}$ & $10 \mathrm{MW}$ \\
MFT operating frequency & $f_{\text {nom }}$ & $250 \mathrm{~Hz}$ \\
Number of MMC cells per branch & $N$ & 22 \\
MMC branch self inductance & $L_{\mathrm{br}}$ & $5 \mathrm{mH}$ \\
MMC branch mutual inductance & $L_{\mathrm{m}}$ & $4.25 \mathrm{mH}$ \\
MMC SM capacitance & $C_{\mathrm{SM}}$ & $5 \mathrm{mF}$ \\
\hline \hline
\end{tabular}

taken into account. With the DR output current mean value being denoted by $I_{\mathrm{R}}$, output voltage of the converter can be calculated according to (10), where $M$ denotes modulation index of both MMCs.

$$
\bar{v}_{\mathrm{o}}=\frac{3}{\pi m_{\mathrm{T} 2}} \underbrace{\left(M \frac{V_{\mathrm{in}}}{2}\right)}_{\text {MMC AC voltage }}-\underbrace{\frac{3}{\pi} \omega L_{\gamma}^{*} I_{\mathrm{R}}}_{\text {Commutation loss }}
$$

Please notice that the higher the equivalent MMC branch inductance the higher the output voltage drop caused by the DR commutation process. However, it can be seen from (4) that branch inductors coupling reduces the equivalent MMC branch inductance, thus decreasing the effect of the DR commutation process.

\section{Control}

System control was organized such that the output voltage mean value follows the reference defined by Table I. Number of SMs per MMC branch was set such that $1.7 \mathrm{kV}$ IGBTs can be used, meaning that the average SM voltage equals $V_{\mathrm{SM}} \approx 900 \mathrm{~V}$. MMC SM capacitance sizing can be conducted following the principles presented in [21], [22]. Therefore, it is determined with the aim of limiting total branch voltage ripple to $\pm 10 \%$ of its nominal value. Finally, MMC branch inductors were designed such that the input current ripple remains within satisfactory limits.

According to Fig. 8, mean value of the DR output voltage is adjusted with the aim of controling the average voltage across the output capacitor $\left(C_{\mathrm{o}}\right)$. Owing to the nature of the output filter, which consists of two reactive elements, two feedback loops are formed, namely outer voltage and inner current loop $\left(W_{\mathrm{R}}\right)$, as presented in Fig. 8 (please notice that all the variables from Fig. 8 denote the average values).

The output voltage is measured, filtered in order to obtain its mean value and compared to the reference set by Table I. Thereafter, voltage error is passed to the PI regulator, whose output represents a desired mean value of the filter inductor $\left(L_{\mathrm{o}}\right)$ current. The filter inductor current is measured, filtered and compared to the reference set by the output voltage regulator. Subsequently, current error is passed to the PI regulator. The converter output current can be used as a feed-forward in case it is measured. Also, the DR commutation voltage drop can be compensated by superimposing $3 \omega L_{\gamma}^{*} I_{\mathrm{R}} / \pi$ to the current PI regulator output. Finally, AC voltage reference for both MMCs is obtained. It is easy to conclude that a variable to be affected by the output voltage control is both MMCs' modulation index $(M)$, which suggest that this converter, in contrast to its bidirectional counter part presented in [10], utilizes the principles of amplitude modulation. To synthesize the desired AC voltage across both MMCs terminals, PhaseShifted Carrier (PSC) modulation is used, although the other modulation methods presented in the literature can as well be employed. It is noteworthy that, similarly to the conventional 3PH DR, dominant harmonics in the filter inductor current, as well as the output voltage occur at six times the frequency of sinusoidal voltage provided by the MMCs, as will be shown in the following section.

Control of the MMC internal quantities, such as the branch energies, can be performed as if conventional grid connected MMC is analyzed. Namely, $\mathrm{T}_{1}$ and $\mathrm{T}_{2} \mathrm{P}$-winding currents can be expanded into Fourier series, as presented in Fig. 9. It can be seen that in either case, transformer current does not contain any even harmonics, nor the ones being the multiples of three. Given that both MMCs supply their associated transformers with sinusoidal voltages (i.e., sine wave at the MFT operating frequency), higher order harmonics in transformers' P-winding currents, do not contribute to the power transfer towards the MMC SMs. As a consequence, circulating currents at the fundamental frequency can be used with the aim of attaining

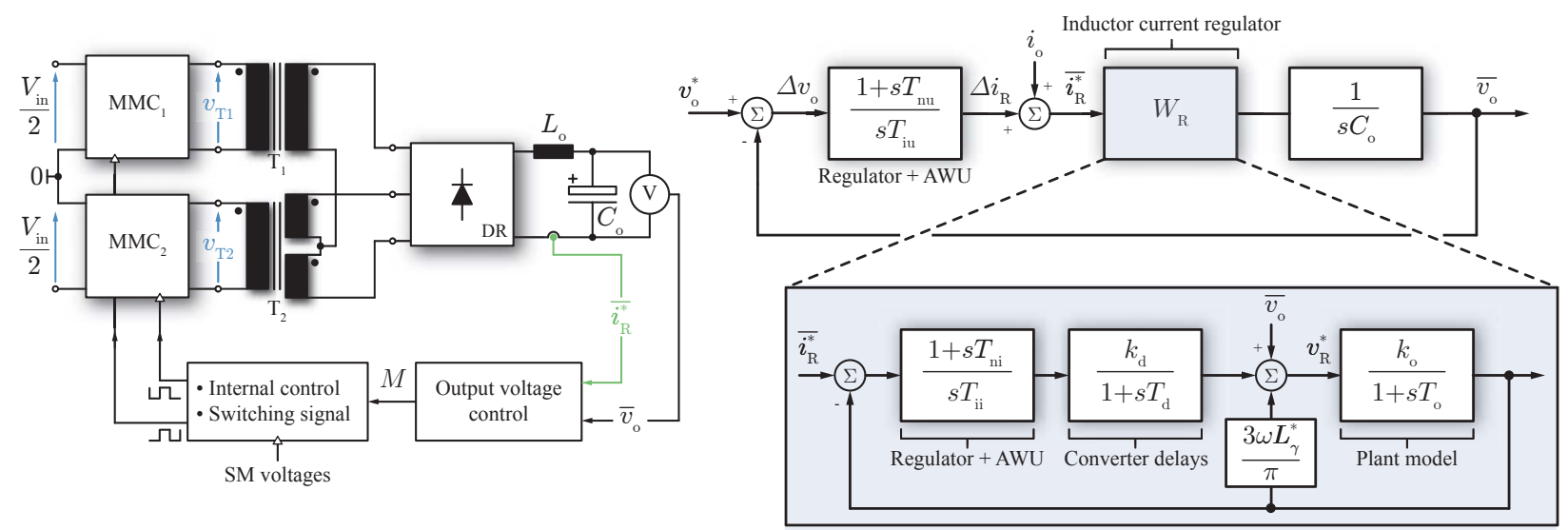

Figure 8: Converter control structure - Two control loops are employed in order to control the output voltage. Parameter to be affected by the control algorithm is both MMCs' modulation index $(M)$. Internal MMC control was organized based on the MMC leg energy levels $\left(W_{\Sigma}, W_{\Delta}\right)$. 


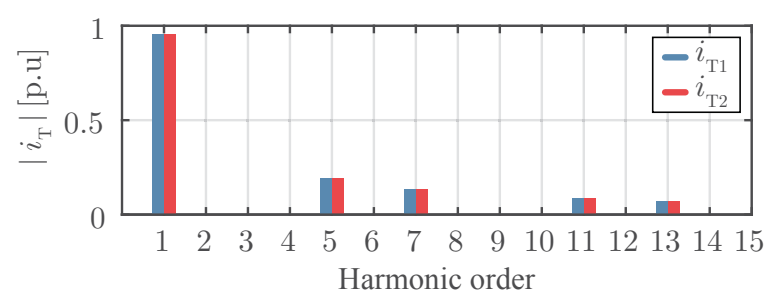

Figure 9: Current spectrum of both transformer units. Frequency axis was normalized with respect to the MFT operating frequency, whereas amplitude axis normalization was performed according to the currents maximum values.

the energy balance within the MMCs [23].

Another conclusion regarding the MMC SMs switching frequency can be made based on Fig. 9. In case PSC modulation is employed, SMs switching frequency should not be set as an integer multiple of the fundamental frequency, as suggested in [24], otherwise side band harmonics in a SM voltage spectrum coincide with the transformers' currents harmonics giving rise to divergence in the MMC branch energies. Hence, SM switching frequency was set as $f_{\mathrm{SW}}=280 \mathrm{~Hz}$.

\section{Simulation Results}

Performance of the proposed converter was investigated using PLECS. Load power profile used with the aim of validating the proposed control scheme can be seen in Fig. 10, whereas parameters of the simulated converter can be found in Table I. Fig. 11 presents converter operation during the time interval of $T_{\text {sim }}=2 \mathrm{~s}$. It can be seen from the bottommost plot that regardless of the power at which the energy is delivered to the load, output voltage follows the defined reference. Moreover, successful tracking of the load power reference can be validated based on the MMCs input currents

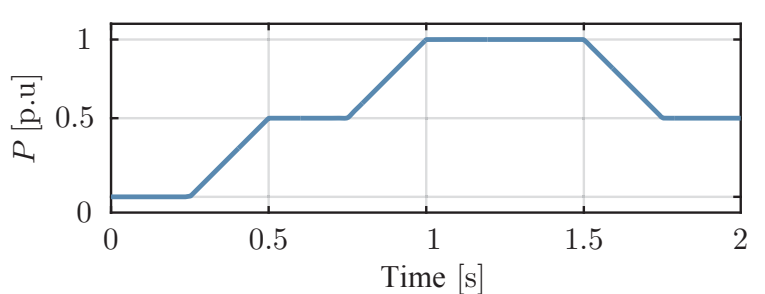

Figure 10: Load power profile used with the aim of validating the proposed control principles. Normalization was performed accroding to the rated power of the converter.

( $i_{\text {in1 }}$ and $i_{\text {in2 }}$, respectively), as well as the DR output current $\left(i_{\mathrm{R}}\right)$, waveforms. Zoomed parts of the graph point out the matching between the simulated waveforms and the idealized ones presented in Fig. 7. Two MMCs generate two quadrature sinusoidal voltages $\left(v_{\mathrm{c}}^{(1)}\right.$ and $v_{\mathrm{c}}^{(2)}$, respectively), resulting in the DR output voltage $\left(v_{\mathrm{R}}\right)$ containing the mean value along with the ripple, occurring at six times converter operating frequency, being superimposed.

For the sake of clarity, Fig. 12 presents converter operation during three fundamental cycles. It can be seen that proposed system retains the properties of both conventional DR and the STC. DR output current $\left(i_{\mathrm{R}}\right)$ consist of the mean value and the higher order harmonics, which can be read from Fig. 13. Frequency axis was normalized with respect to the converter operating frequency. Given that the mean value significantly exceeds the other harmonics being part of the DR output current spectrum, it is presented by the blue bar. The other harmonics, up to the order of fifteen, can be read on the right-hand side axis being colored in red. It is noteworthy that the dominant harmonic in the DR output current occurs at six times operating frequency, as expected according to

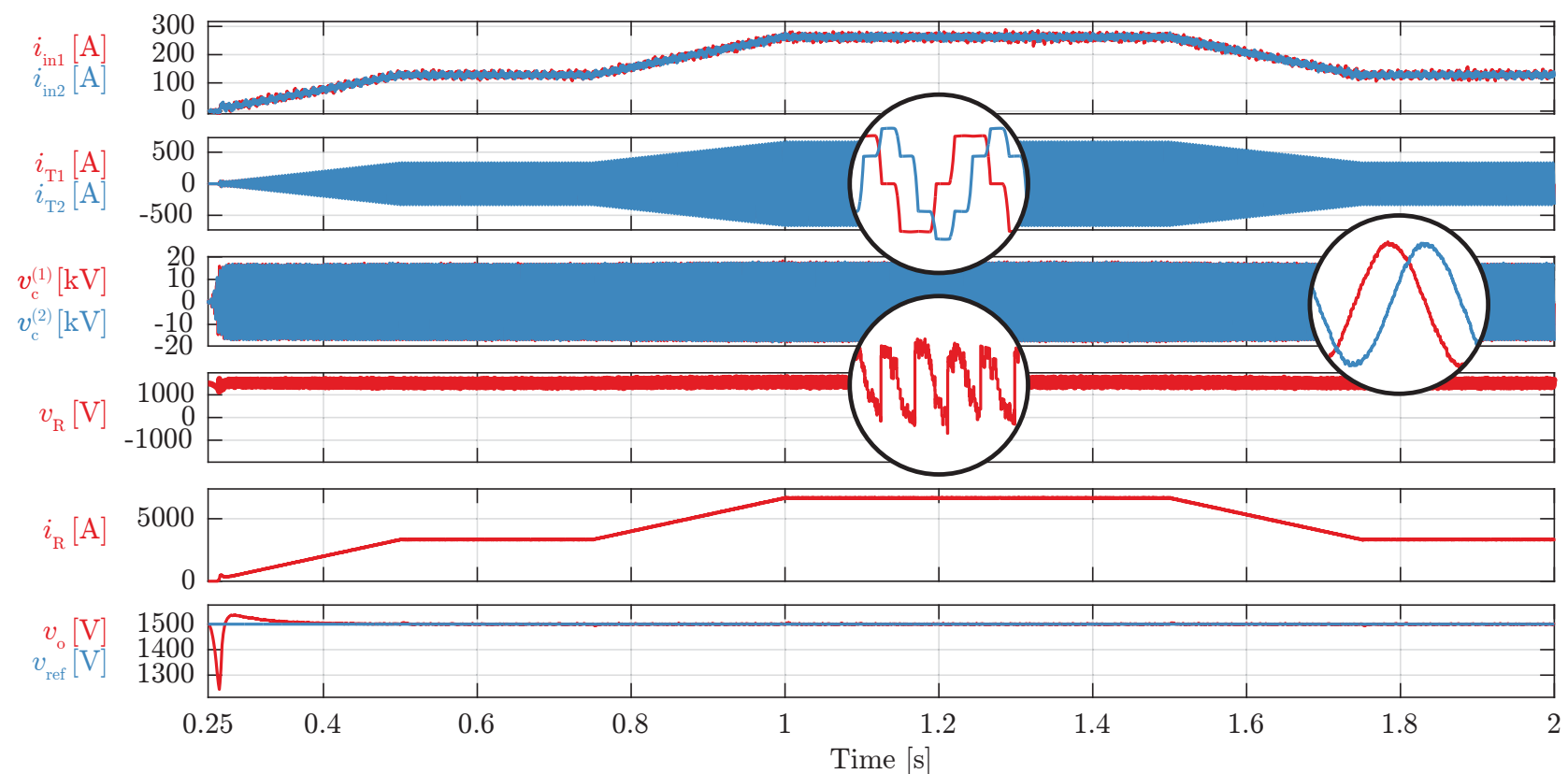

Figure 11: Converter operation during the interval $T_{\mathrm{sim}}=2 \mathrm{~s}$. As can be seen, control system manages to keep the output voltage $\left(v_{o}\right)$ at the desired level. Moreover, load power profile defined in Fig. 10 was successfully tracked, which can be concluded based on the MMCs input currents, as well as the rectifier output current, waveforms. 


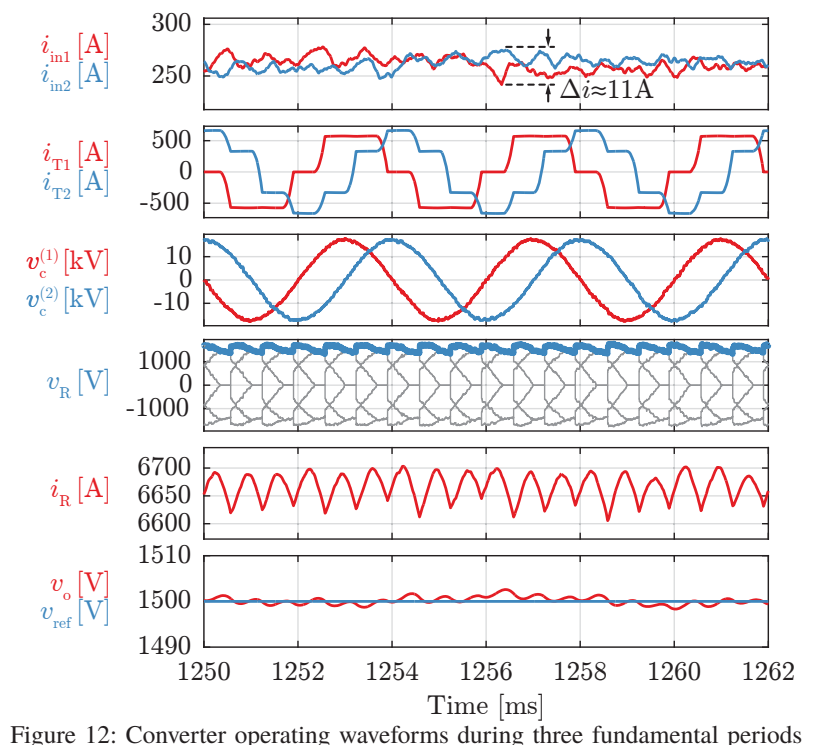

Figure 12: Converter operating waveforms during three fundamental periods

the section on basic operating principles of the proposed topology. Nevertheless, a set of lower order harmonics can be observed. These can be attributed to the nonidealities, which were not taken into account during the discussion on operating principles. For instance, voltages created by the MMCs are not perfectly sinusoidal, which is inevitable no matter which of the modulation schemes is employed, etc. However, harmonics occurring at multiples of six times the converter operating frequency remain the most prominent. Fig. 14 presents both MMCs leg "A" upper branch voltages during three fundamental cycles. Please notice that the voltage ripple across all of the SMs remains within predefined limits.

\section{Conclusion}

This paper presented a novel high-power, unidirectional, DC-DC converter utilizing the STC. Both MMCs employed at the HV/MV stage supply their associated transformers with $1 \mathrm{PH}$, quadrature, sinusoidal voltages. Therefore, seen from the LV stage, which employs conventional 3PH DR, the STC behaves as same as a symmetrical 3PH grid. Considering that converter operation is not constrained to the grid frequency, medium frequency operation of the STC is proposed. Consequently, more compact system can be achieved while maintaining modest switching frequency of the HV/MV stage devices. Operation principles under normal operating conditions were presented and validated through PLECS simulations. In case either of the DC poles is lost, system operation can be maintained though with reduced power, which is a topic to be addressed by the future publications.

\section{ACKNOWLEDGMENT}

This work has been funded in part by the Swiss Competence Center for Energy Research (SCCER FURIES) of the Swiss Innovation Agency Innosuisse and in part by Hyundai Electric and Energy Systems, South Korea.
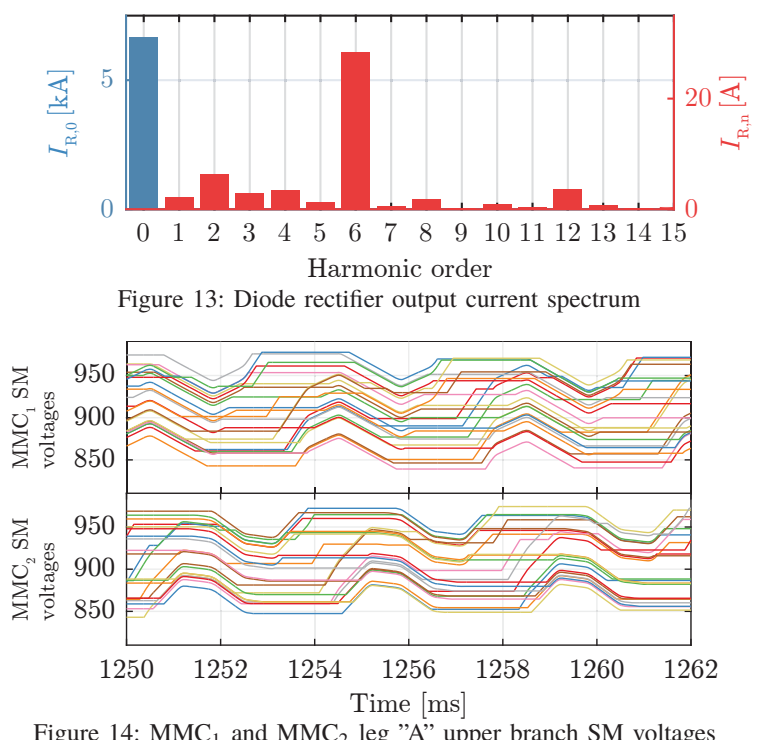

\section{REFERENCES}

[1] A. Clerici, L. Paris, and P. Danfors, "HVDC conversion of HVAC lines to provide substantial power upgrading," IEEE transactions on Power Delivery, vol. 6, no. 1, pp. 324-333, 1991.

[2] M. Häusler, G. Schlayer, and G. Fitterer, "Converting $\mathrm{AC}$ power lines to DC for higher transmission ratings," ABB review, pp. 4-11, 1997.

[3] D. M. Larruskain, I. Zamora, O. Abarrategui, and Z. Aginako, "Conversion of AC distribution lines into DC lines to upgrade transmission capacity," Electric Power Systems Research, vol. 81, no. 7, pp. 1341-1348, 2011.

[4] D. M. Larruskain, I. Zamora, O. Abarrategui, and A. Iturregi, "VSC-HVDC configurations for converting AC distribution lines into DC lines," International Journal of Electrical Power \& Energy Systems, vol. 54, pp. 589597, 2014.

[5] D. Jovcic, "Step-up DC-DC converter for megawatt size applications," IET Power Electronics, vol. 2, no. 6, pp. 675-685, Nov. 2009.

[6] J. A. Ferreira, "The multilevel modular DC converter," IEEE Transactions on Power Electronics, vol. 28, no. 10, pp. 4460-4465, Oct. 2013.

[7] G. J. Kish, M. Ranjram, and P. W. Lehn, "A modular multilevel DC/DC converter with fault blocking capability for HVDC interconnects," IEEE Transactions on Power Electronics, vol. 30, no. 1, pp. 148-162, Jan. 2015.

[8] A. Schön and M. M. Bakran, "A new HVDC-DC converter with inherent fault clearing capability," in 2013 15th European Conference on Power Electronics and Applications (EPE), Sep. 2013, pp. 1-10.

[9] A. Lesnicar and R. Marquardt, "An innovative modular multilevel converter topology suitable for a wide power 
range," in 2003 IEEE Bologna Power Tech Conference Proceedings, vol. 3, Jun. 2003, 6 pp. Vol.3-.

[10] S. Milovanovic and D. Dujic, "MMC-based High Power DC-DC Converter Employing Scott Transformer," in PCIM Europe 2018; International Exhibition and Conference for Power Electronics, Intelligent Motion, Renewable Energy and Energy Management; Proceedings of, Jun. 2018, pp. 226-232.

[11] D. Weiss, M. Vasiladiotis, C. Banceanu, N. Drack, B. Odegard, and A. Grondona, "IGCT based Modular Multilevel Converter for an AC-AC Rail Power Supply," in PCIM Europe 2017; International Exhibition and Conference for Power Electronics, Intelligent Motion, Renewable Energy and Energy Management, May 2017, pp. 1-8.

[12] M. Glinka and R. Marquardt, "A new AC/AC-multilevel converter family applied to a single-phase converter," in The Fifth International Conference on Power Electronics and Drive Systems, 2003. PEDS 2003., vol. 1, Nov. 2003, 16-23 Vol.1.

[13] S. S. et. al., "A modular multilevel converter based Railway Power Conditioner for power balance and harmonic compensation in Scott railway traction system," in 2016 IEEE 8th International Power Electronics and Motion Control Conference (IPEMC-ECCE Asia), May 2016, pp. 2412-2416.

[14] N. D. Yan Zhao and B. Wang, "Application of threephase modular multilevel converter (MMC) in co-phase traction power supply system," in 2014 IEEE Conference and Expo Transportation Electrification AsiaPacific (ITEC Asia-Pacific), Aug. 2014, pp. 1-6.

[15] A. A. Badin and I. Barbi, "Simplified Control Technique for Three-Phase Rectifier PFC Based on the Scott Transformer," in 2006 IEEE International Symposium on Industrial Electronics, vol. 2, Jul. 2006, pp. 931936.

[16] _ _ "Unity Power Factor Isolated Three-Phase Rectifier With Split DC-Bus Based on the Scott Transformer," IEEE Transactions on Power Electronics, vol. 23, no. 3, pp. 1278-1287, May 2008.
[17] _ "Unity Power Factor Isolated Three-Phase Rectifier With Two Single-Phase Buck Rectifiers Based on the Scott Transformer," IEEE Transactions on Power Electronics, vol. 26, no. 9, pp. 2688-2696, Sep. 2011.

[18] J. Tukaram, S. Senthil Kumar, D. Ganesh, and V. Sravan Kumar, "Investigation of PWM current mode controllers for UPF three phase rectifier with split DC bus based on the Scott transformer," in 2012 International Conference on Advances in Power Conversion and Energy Technologies (APCET), Aug. 2012, pp. 16.

[19] R. W. De Doncker, D. M. Divan, and M. H. Kheraluwala, "A three-phase soft-switched high-powerdensity DC/DC converter for high-power applications," IEEE transactions on industry applications, vol. 27, no. 1 , pp. 63-73, 1991

[20] L. Harnefors, S. Norrga, A. Antonopoulos, and H.-P. Nee, "Dynamic modeling of modular multilevel converters," in Power Electronics and Applications (EPE 2011), Proceedings of the 2011-14th European Conference on, IEEE, 2011, pp. 1-10.

[21] M. Vasiladiotis, N. Cherix, and A. Rufer, "Accurate capacitor voltage ripple estimation and current control considerations for Modular Multilevel Converters," IEEE Transactions on Power Electronics, vol. 29, no. 9, pp. 4568-4579, 2014.

[22] K. Ilves, S. Norrga, L. Harnefors, and H.-P. Nee, "On energy storage requirements in modular multilevel converters," IEEE Transactions on Power Electronics, vol. 29, no. 1, pp. 77-88, 2014.

[23] A. Antonopoulos, L. Angquist, and H.-P. Nee, "On dynamics and voltage control of the modular multilevel converter," in Power Electronics and Applications, 2009. EPE'09. 13th European Conference on, IEEE, 2009, pp. 1-10.

[24] K. Ilves, L. Harnefors, S. Norrga, and H. Nee, "Analysis and Operation of Modular Multilevel Converters with Phase-Shifted Carrier PWM," IEEE Transactions on Power Electronics, vol. 30, no. 1, pp. 268-283, Jan. 2015. 